\title{
Radiation Recoil from Highly Distorted Black Holes
}

\author{
Steven Brandt ${ }^{(1,2)}$, Peter Anninos ${ }^{(3,4)}$ \\ (1) Max-Planck-Institut für Gravitationsphysik, Schlaatzweg 1, 14473 Potsdam GERMANY \\ (2) Current address: Pennsylvania State University, 525 Davey Lab, University Park, 16802 \\ (3) National Center for Supercomputing Applications, Beckman Institute, 405 N. Mathews \\ Avenue, Urbana, Illinois, 61801 \\ (4) Current address: University of California, Lawrence Livermore National Laboratory, \\ Livermore, CA 94550
}

(May 8, 2018)

\begin{abstract}
We present results from numerical evolutions of single black holes distorted by axisymmetric, but equatorially asymmetric, gravitational (Brill) waves. Net radiated energies, apparent horizon embeddings, and recoil velocities are shown for a range of Brill wave parameters, including both even and odd parity distortions of Schwarzschild black holes. We find that a wave packet initially concentrated on the black hole throat, a likely model also for highly asymmetric stellar collapse and late stage binary mergers, can generate a maximum recoil velocity of about $150(23) \mathrm{km} / \mathrm{sec}$ for even (odd) parity perturbations, significantly less than that required to eject black holes from galactic cores.
\end{abstract}




\section{INTRODUCTION}

Many popular models of active galactic nuclei, quasars and even archetypical galaxies rely on the relativistic influence of black holes on the surrounding environment to provide power sources for observed spectral emissions and inferred motions of gaseous or stellar material. However, due to the strong gravitational effects of black holes, their role in the evolution of galactic cores and quasars is uncertain since numerical relativity computations are needed to perform detailed investigations of the near-field regime. In particular, gravitational waves generated from sufficiently asymmetric systems (such as collapsing stellar cores and coalescing black holes) can carry a nonzero linear momentum component and impart a recoil velocity to the emitting objects, dominantly from the interplay between mass-quadrupole and mass-octupole or mass quadrupole and current-quadrupole contributions [1,2]. These velocities would be astrophysically significant if they were large enough to eject the emitting objects from the center of the host galaxy and send them hurtling through intergalactic space. Because the efficiency of momentum radiation emission is not known precisely, the dynamics and stability of systems containing black hole engines remain important but unresolved issues. If radiation reaction effects are significant, they may have considerable observable consequences for astrophysics and cosmology, including the redistribution and depletion of black holes from host galaxies, the disruption of active galactic core energetics, the introduction of black holes and stellar material into the intergalactic medium, and the general formation and structure attributes of galaxies.

Although approximation studies of radiation recoil have been performed for more than two decades now, results from these calculations based on quasi-Newtonian and relativistic perturbation formalisms [3 8] present an uncertain picture due to their incomplete treatment. More recently, Anninos and Brandt [9] have numerically computed the recoil effect from fully general relativistic head-on collisions of two unequal mass black holes with timesymmetric initial data, and have shown that recoil velocities are of order $10-20 \mathrm{~km} / \mathrm{sec}$ for black holes with moderately large initial separations ( $\gtrsim 10 M$, where $M$ is the mass of the larger black hole). Their results are in rough agreement with, and generally confirm, various estimates from perturbation calculations.

Here we continue to explore the radiation reaction process by computing the energies and recoil velocities from single black holes distorted by axisymmetric gravitational (Brill [10]) waves. We extend previous Brill wave + black hole investigations [11, 12 by relaxing the equatorial mirror symmetry imposed in earlier work, thus allowing for mixtures of consecutive (even/odd) multipole contributions to the emitted radiation. In addition to investigating radiation reactions in this new class of physical systems, our implementation of Brill waves allows for very highly distorted black holes which can be thought of as models for the late stage behavior of binary coalescing black holes. In fact, we have been able

to simulate single black hole distortions [12,13], as characterized by the ratio of polar to equatorial circumferences of apparent horizons, that are significantly greater than what we have observed in the merged state of two colliding black holes [13 15]. Although the horizon distortion is not the only factor influencing recoil efficiency, one might nevertheless expect to obtain some idea, or perhaps even an absolute upper limit, of the recoil magnitude during the late stages of binary interactions by investigating strongly distorted single black hole systems. 
We generalize the prescription developed in references [11,12] (and summarized for convenience in $\delta[$ (I) to specify equatorially asymmetric initial data and to parameterize Brill wave perturbations of Schwarzschild black holes by the amplitude, shape, location, and spectral mixture of the even and odd $\ell$-modes. Results from numerical evolutions are presented in $\S$ [II, where we show embeddings of the black hole apparent horizons, energies emitted in the most dominant quasinormal modes of the final black hole, and recoil velocities arising from the mixing of consecutive radiative modes. The computations are carried out for both even and odd parity distortions of black holes, and over a wide range of wave strengths, initial placements, and mode distributions. We conclude in $\oint[\mathrm{V}$.

\section{INITIAL DATA}

For even parity distortions, we utilize the conformally flat approach of Bowen \& York [16] to solve the initial value problem in axisymmetry and write the spatial 3-metric at the initial time as

$$
d l^{2}=\Psi^{4}\left[e^{2\left(q-q_{0}\right)}\left(d \eta^{2}+d \theta^{2}\right)+\sin ^{2} \theta d \phi^{2}\right]
$$

where $\Psi(\eta, \theta)$ is the conformal factor, $q(\eta, \theta)$ is a function subject to certain constraints in its form but is otherwise freely specifiable, $q_{0}(\eta, \theta)$ is chosen so that the Kerr metric is recovered if $q=0$ and the appropriate extrinsic curvature is specified, $\eta$ is a logarithmic radial coordinate centered on the black hole throat, and $(\theta, \phi)$ are the usual angular coordinates. The more general 3-metric (applicable to both even and odd parity perturbations) is of the form

$$
\gamma_{i j}=\Psi^{4}\left[\begin{array}{ccc}
A(\eta, \theta) & 0 & 0 \\
0 & B(\eta, \theta) & F(\eta, \theta) \sin \theta \\
0 & F(\eta, \theta) \sin \theta & D(\eta, \theta) \sin ^{2} \theta
\end{array}\right]
$$

with $F=0(\neq 0)$ for the even (odd) parity cases. The remaining metric components $\left(\gamma_{\eta \theta}\right.$ and $\left.\gamma_{\eta \phi}\right)$ are set to zero by the gauge freedom in choosing the shift vector.

As in Ref. [12], the somewhat arbitrary function $q$ is restricted by symmetry conditions on the throat and axis, and fall-off rates at large radii [11]. The function $q$ is constructed to have an inversion symmetric Gaussian part given by

$$
q=Q_{0} f(\theta)\left(e^{-s_{+}}+e^{-s_{-}}\right)+q_{1}
$$

where

$$
s_{ \pm}=\frac{\left(\eta \pm \eta_{0}\right)^{2}}{\sigma^{2}}
$$

and $q_{1}=0$ or $q_{0}$ for perturbations of the stationary Kerr solution or the Bowen \& York spacetime [16 respectively. With this form, the Brill waves are characterized by their amplitude $Q_{0}$, width $\sigma$, center coordinate location $\eta_{0}$, and their angular dependence $f(\theta)$. This allows a convenient way to parameterize the strength, shape and placement of the waves, and to easily tune the wave data for a broad range of spectral mode mixtures. 
In previous work, we had considered the case $f=\sin ^{n} \theta$ which possesses mirror symmetry across the equator. However, this data does not allow for the emitted gravitational waves to carry any linear momentum, as it excludes odd multipole components. Here, we relax the constraint of equatorial symmetry and consider

$$
f(n, \xi, \theta)=(1-\xi+\xi \cos \theta) \sin ^{n} \theta,
$$

for which $q$ in (3) obeys the isometry conditions $(\eta \rightarrow-\eta, \theta \rightarrow-\theta$, and $\theta \rightarrow 2 \pi-\theta)$. The form of (5) also has the necessary property $f(0)=f(\pi)=0$, and regulates the even and odd mode power distributions through the parameters $n$ and $\xi$. The parameter $\xi$ determines the asymmetry of the wave, and the relative excitation of the odd and even numbered, even parity $\ell$ modes. When $\xi=0$ and $n=2, \ell=2$ is the dominant mode; when $\xi=1$ and $n=2$, $\ell=3$ is the dominant mode. For some intermediate value of $\xi$ there will be a roughly even distribution of energy between the $\ell=2$ and $\ell=3$ modes, and at this value the gravity waves will produce a maximum recoil velocity on the black hole, as demonstrated in $\delta[1]$. The initial value problem for the even parity cases is completed by solving the Hamiltonian constraint for the conformal factor $\Psi$ in the metric (1) with the specified free data. We also impose time symmetry, hence the extrinsic curvature is set to zero and the momentum constraint is trivially satisfied. This implies that the Brill wave packet is a combination of ingoing and outgoing radiation.

For odd parity distortions, the free data is specified in the only nontrivial momentum constraint equation arising from the $\phi$ component in "time-rotation" symmetry and maximal slicing $(\operatorname{tr} K=0)$. Defining the initial extrinsic curvature as

$$
K_{i j}=\Psi^{-2}\left[\begin{array}{ccc}
0 & 0 & \hat{H}_{E} \sin ^{2} \theta \\
0 & 0 & \hat{H}_{F} \sin \theta \\
\hat{H}_{E} \sin ^{2} \theta & \hat{H}_{F} \sin \theta & 0
\end{array}\right],
$$

the momentum constraint reduces to

$$
\partial_{\eta}\left(\hat{H}_{E} \sin ^{3} \theta\right)+\partial_{\theta}\left(\hat{H}_{F} \sin ^{2} \theta\right)=0,
$$

and is satisfied by

$$
\begin{aligned}
& \hat{H}_{E}=f_{1}(\theta)+f_{2}(\eta)\left[4 \cos \theta f_{3}(\theta)+\sin \theta \partial_{\theta} f_{3}(\theta)\right], \\
& \hat{H}_{F}=-\partial_{\eta} f_{2}(\eta) \sin ^{2} \theta f_{3}(\theta),
\end{aligned}
$$

where $f_{1}, f_{2}$ and $f_{3}$ are arbitrary functions with the following symmetries: $f_{1}(\theta)=f_{1}(-\theta)=$ $f_{1}(2 \pi-\theta), f_{3}(\theta)=f_{3}(-\theta)=f_{3}(2 \pi-\theta), f_{2}(\eta)=f_{2}(-\eta)$, and $f_{2} \rightarrow 0$ as $\eta \rightarrow \infty$ so the spacetime asymptotically approaches the Kerr solution. To construct a model for odd parity waves analogous to the even parity case described above, we choose the following free functions:

$$
\begin{aligned}
& f_{1}=0, \\
& f_{2}=Q_{0}\left(e^{-s_{+}}+e^{-s_{-}}\right), \\
& f_{3}=(1-\xi+\xi \cos \theta) \sin ^{n} \theta .
\end{aligned}
$$

Since $\hat{H}_{E}$ falls off sufficiently rapidly at large radii, any spacetime constructed using this conformal extrinsic curvature with $f_{1}=0$ will have zero angular momentum. The Hamiltonian constraint is then solved for $\Psi$ given the above extrinsic curvature and a conformally flat 3-metric with $q=q_{0}=0$ in (四). 


\section{RESULTS}

In this section we present results from several dozen numerical calculations of both even and odd parity axisymmetric distortions of single Schwarzschild black holes using the initial data parameterization described in $\S[1]$. The results are presented as functions of various key Brill wave parameters, and their effect on radiation recoil is evaluated. In most cases we have used a numerical grid with $300 \times 65$ (radial $\times$ angular) zones to cover radial distances out to several hundred $M_{A D M}$, where $M_{A D M}$ is the ADM mass of the spacetime, and to include the entire polar domain $0 \leq \theta \leq \pi$. However, we have also confirmed that the results are robust and relatively unchanged at different grid resolutions. Our simulations utilize the maximal slicing condition $(K=\dot{K}=0)$ and are generally run to $50-70 M_{A D M}$, which is more than enough time to extract the radiation content. The radiated wave energies and recoil velocities are computed from the energy-momentum flux across a spherical shell of radius $15 M_{A D M}$ from the center of the black hole throat.

\section{A. Even Parity}

First we consider the effects of varying three independent parameters $Q_{0}, \eta_{0}$ and $\xi$, corresponding to the amplitude, peak location, and dominant mode of the Brill waves, on the radiation reaction and dynamical evolution of even parity distortions of black holes. The remaining free initial data parameters described in $\S[\mathbb{I}$ have been held fixed: $\sigma=1$ for unit width wave profiles, $q_{0}=0$ since we do not consider rotating black holes, and $n=2$ to allow maximum grid resolution over the angular variations.

From the equatorially symmetric examples in Refs. [11-13], it is known that increasing the amplitude parameter $Q_{0}$ increases the strong field coupling of the Brill wave and black hole, substantially distorts the spacetime from spherical symmetry, and emits a greater fraction of the ADM mass in the form of gravitational radiation. To demonstrate the degree by which a black hole is distorted from sphericity, we first look at the geometric characteristics of the spacetime, namely the apparent horizon since it can easily be found in the spacelike slices. The horizon shape parameters and flat space embeddings are evaluated for the case $\eta_{0}=0$ in which the Brill wave is placed directly on the black hole throat for maximum horizon distortion. We use a Newton-Raphson [17, 18 procedure to solve the nonlinear equation defining the trapped surface conditions (zero expansion of outgoing null normals to the 2-surface). The geometric properties are extracted from the two-dimensional sub-metric induced on the horizon surface

$$
d l^{2}=\Psi^{4}\left\{\left[B+A\left(\frac{d h}{d \theta}\right)^{2}\right] d \theta^{2}+D d \phi^{2}+2 F d \theta d \phi\right\},
$$

where $h(\theta)$ is the radial coordinate defining the horizon. Visual representations of the horizon are achieved by embedding the 2-surface given by (13) in a higher three-dimensional flat space. Introducing a new coordinate $z$ on a flat 3 -metric, the $2-$ metric of the horizon surface is identified as

$$
d z^{2}+d \rho^{2}+\rho^{2} d \phi^{2}=B^{\prime}(\theta) d \theta^{2}+D^{\prime}(\theta) d \phi^{2}
$$


where $B^{\prime}=\Psi^{4}\left(B+A(d h / d \theta)^{2}+F^{2} / D\right)$ and $D^{\prime}=\Psi^{4} D$ are the metric components of the horizon surface transformed to a diagonal form. Solving for the coordinate $z$ gives

$$
z=\int d \theta \sqrt{B^{\prime}-\left(\partial_{\theta} \sqrt{D^{\prime}}\right)^{2}}
$$

which is integrated numerically to obtain the embedding functions $z(\theta)$ and $\rho(\theta)=\sqrt{D^{\prime}}$, although an embedding is not in general guaranteed to exist.

In figure 11 we show embeddings of the horizon 2-surface in the initial data (where distortions are greatest with $\eta_{0}=0$ ) for a highly perturbed $Q_{0}=0.9$ case with different mode parameters $\xi$. Embeddings of the more prolate odd mode distortions, i.e. $\xi \geq 0.7$, are undefined (and therefore not displayed) for the more negative values of $z$ due to the radical in equation (15) which becomes negative. Figure 2 shows the equivalent embeddings for $\xi=0.5$ as a function of wave amplitude $Q_{0}$. These embedded distortions eventually damp out in an oscillatory fashion over time as the horizon evolves towards sphericity after the dynamic component has either been absorbed by the black hole, or has propagated to asymptotic infinity in the form of gravitational waves. Together, the embedding diagrams indicate that horizon distortions are roughly spherical for small amplitude perturbations and become generally more prolate as $Q_{0}$ is increased. The shape of distortions also varies with the mode parameter $\xi$, which regulates changes from equatorially symmetric even mode behavior, to predominantly asymmetric odd mode configurations for the larger values of $\xi$. The ratio of polar to equatorial circumferences of the horizon surfaces, used in our previous work as a measure of distortion, is not especially informative regarding the magnitude of radiation recoil. Indeed, the purely even symmetric cases generally give rise to greater distortions, but no recoil which is a function of the relative mixture of even and odd modes, as well as the perturbation amplitude. Assuming a simple definition of radial distortion in the embeddings as $R_{r}=\max \left(\sqrt{\rho^{2}+z^{2}}\right) / \min \left(\sqrt{\rho^{2}+z^{2}}\right)$, the displayed distortions range from $R_{r}=1.2$ for $\left(Q_{0}=0.1, \xi=0.9\right)$, to 7.5 for $\left(Q_{0}=1.2, \xi=0.1\right)$.

The transition from even to odd mode behavior observed in the near-field horizon embedding diagrams of figure [1, is also mirrored by the mode distribution in the far-field radiation zone. Figures 3 and 1 show the energy (normalized to the ADM mass of the spacetime) radiated in the most dominant $\ell=2$ and 3 modes for $\eta_{0}=0$ as a function of $\xi$ and $Q_{0}$. As the mode parameter is increased in figure 3, the energy distribution dominance changes from even to odd, consistent with the horizon embeddings in figure 11. Figure 4 indicates that the total radiated energies asymptotically approach constant maximal values for each of the mode parameters, and that the range of parameters we have investigated are reasonably representative of the most efficient radiators of gravitational energy. We have restricted current studies to wave amplitudes $Q_{0} \leq 1.2$, since the numerical results are less reliable for larger amplitudes, especially at late times and in the ability to resolve both the $\ell=2$ and 3 modes in the extreme odd or even $\ell$-mode dominated evolutions.

The mixing of adjacent multipole modes gives rise to a non-vanishing flux of linear momentum along the $z$-axis which can be evaluated from products of consecutive Zerilli wave functions [8]

$$
\frac{d P^{z}}{d t}=\frac{1}{16 \pi} \sum_{l=2}^{\infty} \sqrt{\frac{(\ell-1)(\ell+3)}{(2 \ell+1)(2 \ell+3)}} \frac{d \psi_{\ell}}{d t} \frac{d \psi_{\ell+1}}{d t}
$$


where the Zerilli functions $\psi_{\ell}$ are normalized such that the total radiated energy in each mode is given by

$$
E_{\ell}=\frac{1}{32 \pi} \int d t\left(\dot{\psi}_{\ell}\right)^{2}
$$

For numerically practical purposes, we compute only the most significant $(2,3)$ and $(3,4)$ contributions. In general we find that the higher order terms in the series (16) play an increasingly greater role as $\xi$ is increased and as the distortions become dominantly odd functions. For an interesting large amplitude case $\left(Q_{0}=0.9\right)$ the momentum ratio $P_{(2,3)} / P_{(3,4)}$ varies from roughly 50 to 0.2 for $\xi=0.1$ and 0.9 respectively, with even greater ratios for the smaller amplitude cases. However, as we show below, the greatest recoil velocities arise for roughly equal mixtures of even and odd perturbations (i.e., $\xi=0.5$ ), and for these cases the $(2,3)$ contribution exceeds the $(3,4)$ by at least a factor of 50 in all cases we have studied. The results presented for the radiated momentum are derived by summing both the $(2,3)$ and $(3,4)$ contributions. To confirm the degree to which these two dominant pairs are complete, and to independently check our calculations, we have also evaluated the momentum flux from the Landau-Lifshitz pseudotensor [19 21]

$$
\frac{d P^{z}}{d t}=\frac{r^{2}}{16 \pi} \int\left[(\dot{F})^{2}+\frac{1}{4}(\dot{B}-\dot{D})^{2}\right] \cos \theta d \Omega,
$$

which is valid for both even $(F=0)$ and odd $(B=D=0)$ parity perturbations. We find excellent agreement, typically better than $10 \%$, between the two calculations.

As a result of the momentum emission from the interplay between even and odd $\ell$-modes, the final black hole will acquire a recoil velocity

$$
v_{r}^{z}=-\frac{1}{M_{A D M}} \int\left(\frac{d P^{z}}{d t}\right) d t
$$

that is opposite in direction to the momentum flux of the waves. We show these velocities in figures 5 and 6 in physical units of kilometers per second, and as a function of the parameters $\xi, \eta_{0}$ and $Q_{0}$. Figure 5 confirms that maximum recoil occurs at $\xi=0.5$. The asymptotic flatness of the curves at large values of $Q_{0}$ in figure 6 suggests maximum velocities of about 150,200 , and $500 \mathrm{~km} / \mathrm{sec}$ for the most strongly distorted cases that we are able to compute numerically for $\eta_{0}=0,0.25$ and 0.5 respectively. However, we are unable to reliably evolve greater amplitudes for $\eta_{0}=0.25$ or 0.5 and establish a precise turnover velocity since our code breaks at these large amplitudes, though the curves already show signs of flattening by $Q_{0}=1.2$. Hence the quoted values in these two cases are approximate extrapolations. Also notice that greater recoil velocities result when the Brill waves are placed at larger radii, beyond the perturbation potential barrier (at $r>3 M$, or equivalently $\eta>1.8$ ). In these cases, the ingoing waves excite the ringing modes more strongly as they cross the barrier and emit a greater flux of energy-momentum. On the other hand, it is also likely that a large fraction of the radiated flux in the large $\eta_{0}$ cases can be attributed to the outgoing wave component and general distortions of the global spacetime [18], and not to pure black hole ringing from localized collapse or impact scenarios. 


\section{B. Odd Parity}

Following the general presentation of $\oint \overline{\Pi \Pi A}$, we present in this section recoil velocities from odd parity distortions of black holes as a function of mode parameter $(\xi)$, initial peak location $\left(\eta_{0}\right)$, and wave amplitude $\left(Q_{0}\right)$ in the initial data of $\$$ [I].

The momentum arising from consecutive $\ell$-mode interactions of odd parity waves takes a form analogous to (16), except the wave functions $\psi_{\ell}^{\text {odd }}$ (replacing the even parity $\dot{\psi}_{\ell}$ ) are extracted from the $\gamma_{\theta \phi}$ metric component by

$$
\psi_{\ell}^{\text {odd }}=\sqrt{\frac{2(\ell-2) !}{(\ell+2) !}} \int \partial_{\eta} F\left(\partial_{\theta}^{2}-\cot \theta \partial_{\theta}\right) Y_{\ell 0} d \Omega .
$$

In this form, the wave functions are related to the Regge-Wheeler [22] perturbation variable $h_{2}$

$$
\psi_{\ell}^{\text {odd }}=\sqrt{\frac{(\ell+2) !}{2(\ell-2) !}} r \partial_{r}\left(\frac{h_{2}}{r^{2}}\right)
$$

with normalization

$$
E_{\ell}=\frac{1}{32 \pi} \int d t\left(\psi_{\ell}^{o d d}\right)^{2}
$$

An important difference in the evolutions of odd (versus even) parity data is that it is necessary to keep track of the momentum contributions from a greater number of $\ell-$ mode pairs since there is no clear dominance by the lowest order terms. The individual contributions can jump from positive to negative values of momentum with roughly the same amplitude over consecutive pairs. For example, the better behaved $\eta_{0}=0$ small amplitude cases require at least three pairs in the series to converge at the $10 \%$ level when compared with subsequent additions and to the Landau-Lifshitz formula (18). Also, the odd parity initial value problem and the dynamical evolutions over time can both generate significant even parity signals, contributing up to a few percent of the net recoil velocity in the cases we have investigated. Hence all results in this section are derived from the Landau-Lifshitz pseudotensor, thus accounting for all the modes in evaluating the velocities (though we have compared and confirmed the consistency of both methods for small and large amplitude runs). We plot in Figures 6 and 8 the velocities as a function of $\xi$ and $Q_{0}$ respectively. Figure 7 indicates that maximum recoil is achieved for $\xi \approx 0.7$. Figure 8 shows the maximum velocity for $\xi=0.7$ as $Q_{0}$ is varied over a numerically robust range of amplitudes for three different initial wave positions $\eta_{0}=0,0.5$ and 1 . The evolutions generate maximum velocities of 23, 52 and $430 \mathrm{~km} / \mathrm{sec}$, with increasingly greater velocities for Brill waves initially concentrated at greater distances from the black holes.

In comparing these results with the even parity cases (say $\eta_{0}=0$ ), one should not necessarily conclude that odd parity radiation is less effective in producing radiation recoil for any intrinsic reason. Much has to do with the manner in which the data was constructed. For example, the even parity data distorts the spatial metric, while the odd parity data uses a conformally flat metric. One could, in principle, produce even parity distortions of the spacetime through the extrinsic curvature while maintaining a flat metric. Likewise, one 
could add odd parity data to the metric itself rather than the extrinsic curvature. This would make the procedures more similar (and possibly the radiation energies as well). Furthermore, there are many ways to construct initial data for both types of radiation and it is not feasible to study them all. Rather, our results represent the maximal effects of a certain class of black hole distortions.

\section{CONCLUSION}

We have carried out a systematic study of single black holes distorted by strong-field axisymmetric Brill waves in an effort to quantify the astrophysical significance of the "rocket" effect imparted to the final black hole from the momentum carried by gravitational radiation in the system. This work compliments our previous studies of the head-on collision of two unequal mass black holes [9], where we found recoil velocities up to $10-20 \mathrm{~km} / \mathrm{sec}$. However, it is likely that coalescing binary black holes with arbitrary physical parameters (i.e., impact parameters, masses, and spins) may generate greater recoil velocities, so we have focused these current studies to deduce the maximum recoil expected from highly asymmetrical configurations. The Brill wave + black hole systems we have studied allow a parameterization of the wave strengths, widths, locations, and shapes of the perturbing sources such that we are able to systematically explore the role of various parameters in fully nonlinear numerical calculations of strongly distorted black hole spacetimes. With this approach, we are able to generate greater distortions and wider spectral energy distributions of black holes than observed in our simulations of colliding binary systems. We thus also consider our current results as reasonable maximum estimates of radiation recoil in single or late-stage binary black hole systems (although a more precise comparison between single and binary evolutions must also account for any residual radiation content in the initial data of the respective systems).

For the most highly distorted spacetimes, we find maximum recoil velocities in excess of $400 \mathrm{~km} / \mathrm{sec}$ for both even and odd parity data with Brill waves initially centered at large distances from the black hole throat, e.g., $\eta_{0}=0.5$ (1.0) for even (odd) parity perturbations. Our results exhibit a strong dependence on the initial placement of the Brill waves, as well as their amplitude and spectral composition. Of all these effects, we are less certain of the role which the initial wave placement $\eta_{0}$ plays in generating a true maximum value, since for the numerically difficult combination of large separations and amplitudes, our code eventually breaks down. However, we expect for radiation clumps located further from the black hole, that a substantial fraction of the (outgoing component of the) Brill waves escapes to infinity since the perturbations are applied essentially to the spacetime surrounding the black hole, and not directly on the throat. Hence we expect that the bulk of emitted energymomentum flux can be attributed to the initial wave configuration for large $\eta_{0}$, as opposed to any intrinsic ringing of the black hole associated with localized source dynamics such as ingoing wave collisions, collapsing stellar cores, or coalescing binaries. On the other hand, an ingoing wave located outside the potential barrier can scatter off and impart a much greater momentum to the hole. We were, however, unable to distinguish secondary wave pulses in our numerical data corresponding to reflected waves.

In addition to reducing the initial outgoing Brill wave content, it is also likely that the $\eta_{0}=0$ cases represent more appropriate late stage recoil models for black hole binary sys- 
tems. In these cases, our results of 150 and $23 \mathrm{~km} / \mathrm{sec}$ for even and odd parity perturbations are in general agreement with the bound $v_{r} \leq 300 \mathrm{~km} / \mathrm{sec}$ derived by Bekenstein [3] in his quasi-Newtonian considerations of the interaction between quadrupole and octupole terms in non-spherical stellar core collapse to black holes. Furthermore, the odd parity recoil in our calculations is remarkably similar to the $25 \mathrm{~km} / \mathrm{sec}$ found by Moncrief [ [I] for non-spherical models of black hole formation. Our even parity results are approximately a factor of two times larger than the quasi-Newtonian calculations $\left(v_{r} \sim 67 \mathrm{~km} / \mathrm{sec}\right)$ of binary systems in Keplerian orbits by Fitchett [5]. However, considering the ambiguity in choosing the final prior-to-plunging orbit and in extrapolating the perturbation calculations to the equal mass limit, our results are in fairly good agreement with the predictions of Fitchett and Detweiler [6] who extended Fitchett's earlier work to perturbation theory and computed a maximum velocity of about $120 \mathrm{~km} / \mathrm{sec}$ for the merging of two black holes from the last stable circular orbit. We are also in agreement with Nakamura, Oohara and Kojima [7] who estimate a maximum velocity of about $240 \mathrm{~km} / \mathrm{sec}$ from numerical perturbative calculations of test bodies plunging into black holes from infinity with arbitrary orbital angular momentum.

In comparison, the escape velocity from galactic structures can vary from about several hundred $\mathrm{km} / \mathrm{sec}$ for spiral galaxies such as the Milky Way, to about a thousand $\mathrm{km} / \mathrm{sec}$ for the more massive giant ellipticals such as M87. Our results, however, suggest that black holes which may be located in the centers of galaxies and which undergo highly asymmetric evolutions (including strong field distortions and binary mergers) are relatively stable entities and will not likely escape from the host galaxy, assuming that the "on the throat" numerical calculations are reasonably representative models. Although we have established that the recoil effect is not generally large enough to be considered astrophysically significant, this does not, however, rule out the possibility of black hole ejections from galactic disks far from the core and in the direction of galactic rotation, nor the possibility of black hole ejections from globular cluster systems in galactic halos. Black holes can more readily escape from these systems to wander through the galaxy or even intergalactic space.

\section{ACKNOWLEDGMENTS}

The numerical simulations were performed on the Origin2000 machines at the NCSA and the Albert Einstein Institute. This work was supported by NSF grant PHY 98-00973, and performed under the auspices of the U.S. Department of Energy by Lawrence Livermore National Laboratory under Contract W-7405-Eng-48. 


\section{REFERENCES}

[1] Bonnor and Rotenberg, Proc. Roy. Soc. A265, 109 (1961)

[2] A. Perez, Phys. Rev. 128, 2471 (1962)

[3] J.D. Bekenstein, Astrophys. J. 183, 657 (1973)

[4] V. Moncrief, Astrophys. J. 238, 333 (1980)

[5] M.J. Fitchett, MNRAS 203, 1049 (1983)

[6] M.J. Fitchett and S. Detweiler, MNRAS 211, 933 (1984)

[7] Nakamura, Oohara and Kojima, Prog. Theor. Phys. Suppl. 90, 135 (1987)

[8] Z. Andrade and R.H. Price, Phys. Rev. D, 56, 6336 (1997)

[9] P. Anninos and S. Brandt, Phys. Rev. Lett., 81, 508 (1998)

[10] D.S. Brill, Ann. Phys. (N.Y.) 7, 466 (1959)

[11] D. Bernstein, D. Hobill, E. Seidel and L. Smarr, Phys. Rev. D, 50, 3760 (1994)

[12] S. Brandt and E. Seidel, Phys. Rev. D, 54, 1403 (1996)

[13] P. Anninos, D. Bernstein, S. Brandt, D. Hobill, E. Seidel and L. Smarr, Phys. Rev. D, 50, $3801(1994)$

[14] P. Anninos, D. Hobill, E. Seidel, L. Smarr and W.M. Suen, Phys. Rev. Lett., 71, 2851 (1993)

[15] P. Anninos, D. Hobill, E. Seidel, L. Smarr and W.M. Suen, Phys. Rev. D, 52, 2044 (1995)

[16] J. Bowen and J.W. York, Phys. Rev. D, 21, 2047 (1980)

[17] G. Cook, Ph.D. thesis, University of North Carolina at Chapel Hill, Chapel Hill, North Carolina (1990)

[18] D. Bernstein, Ph.D. thesis, University of Illinois, Urbana-Champaign (1993)

[19] Landau, L.D. and Lifshitz, E.M., The Classical Theory of Fields, (Pergamon Press, 1975)

[20] Cunningham, C.T., Price, R.H. and Moncrief, V., Astrophys. J. 224, 643 (1978)

[21] Cunningham, C.T., Price, R.H. and Moncrief, V., Astrophys. J. 230, 870 (1979)

[22] T. Regge and J. Wheeler, Phys. Rev. 108, 1063 (1957) 


\section{FIGURES}

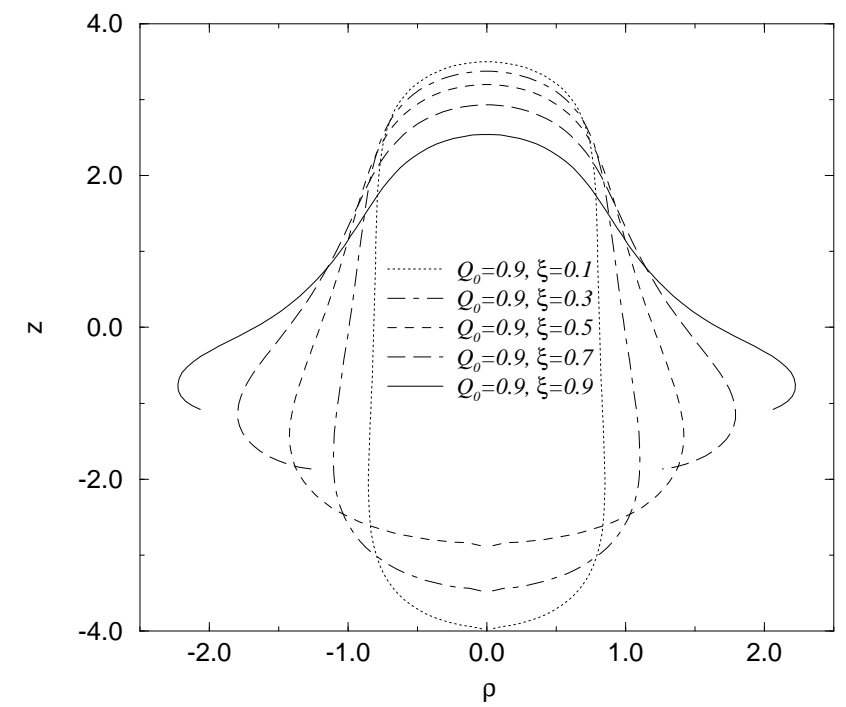

FIG. 1. Embeddings in 3-dimensional flat space of the apparent horizon 2-surfaces at the initial time for even parity perturbations. Each curve represents the $\rho-z$ embedding for the same strongly distorted case with Brill wave amplitude $Q_{0}=0.9$ and wave location $\eta_{0}=0$, but with different mode parameters $\xi$ that regulate the relative mixture of even and odd mode spectral distributions. Distortions range from $R_{r}=1.9$ for the $\xi=0.9$ case to $R_{r}=4.9$ for $\xi=0.1$, where $R_{r}=\max \left(\sqrt{\rho^{2}+z^{2}}\right) / \min \left(\sqrt{\rho^{2}+z^{2}}\right)$ is the ratio of the maximum to minimum distances of the embedding curves to the origin at $\rho=z=0$.

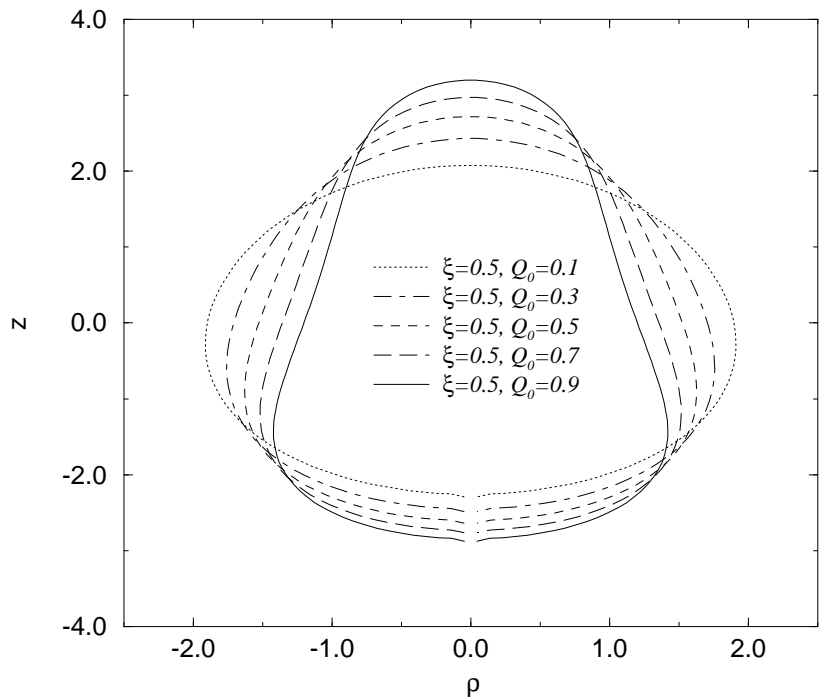

FIG. 2. As figure 1, except here each curve represents the embedding for the same mode parameter $\xi=0.5$ which generates the greatest recoil effect for a specified amplitude $Q_{0}$. Distortions range from $R_{r}=1.2$ for $Q_{0}=0.1$ to $R_{r}=2.7$ for $Q_{0}=0.9$. 


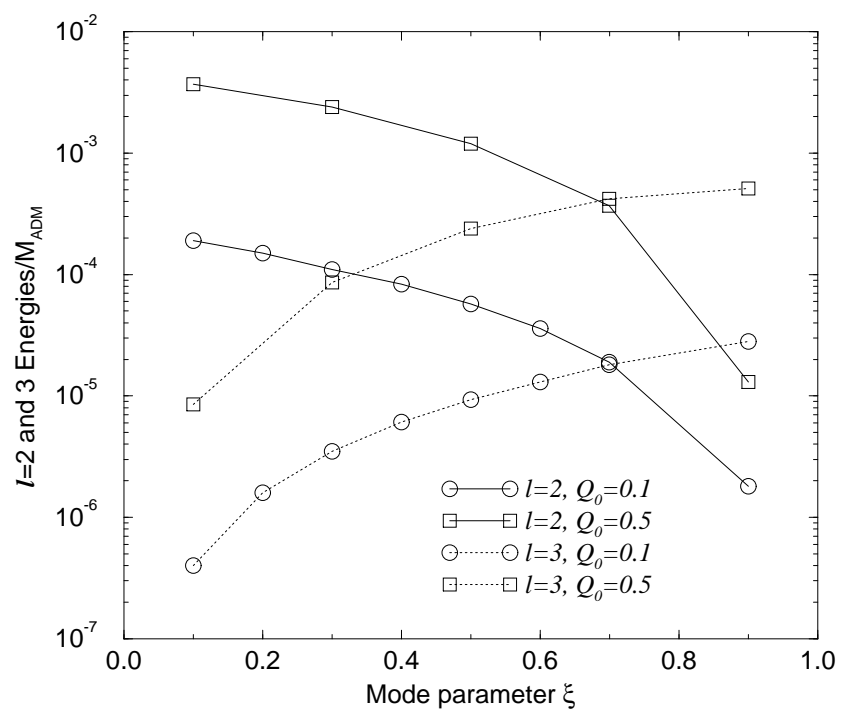

FIG. 3. Radiated energies (normalized to the ADM mass, $M_{A D M}$ ) in the most dominant $\ell=2$ and 3 even parity modes as a function of the Brill wave mode parameter $\xi$ for $\eta_{0}=0$ and select values of $Q_{0}$. The wave extractions are performed on spherical shells of radius $15 M_{A D M}$ and centered on the black hole throat.

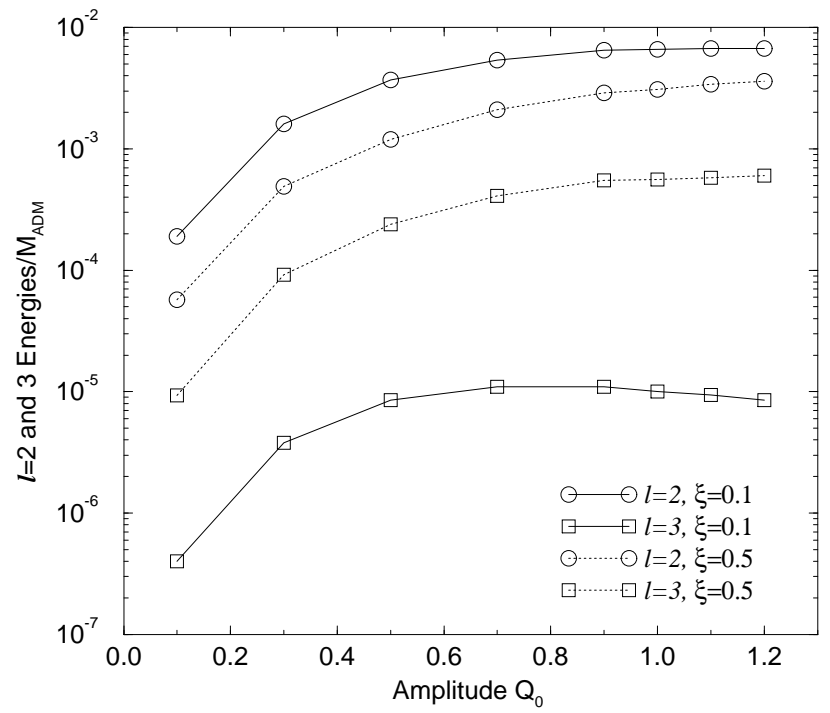

FIG. 4. As figure 3, except the energies are plotted as a function of Brill wave amplitude for select values of $\xi$. The upper bound on the wave amplitudes considered here is dictated by the accuracy with which the weaker of the $\ell=2$ or 3 modes can be resolved. 


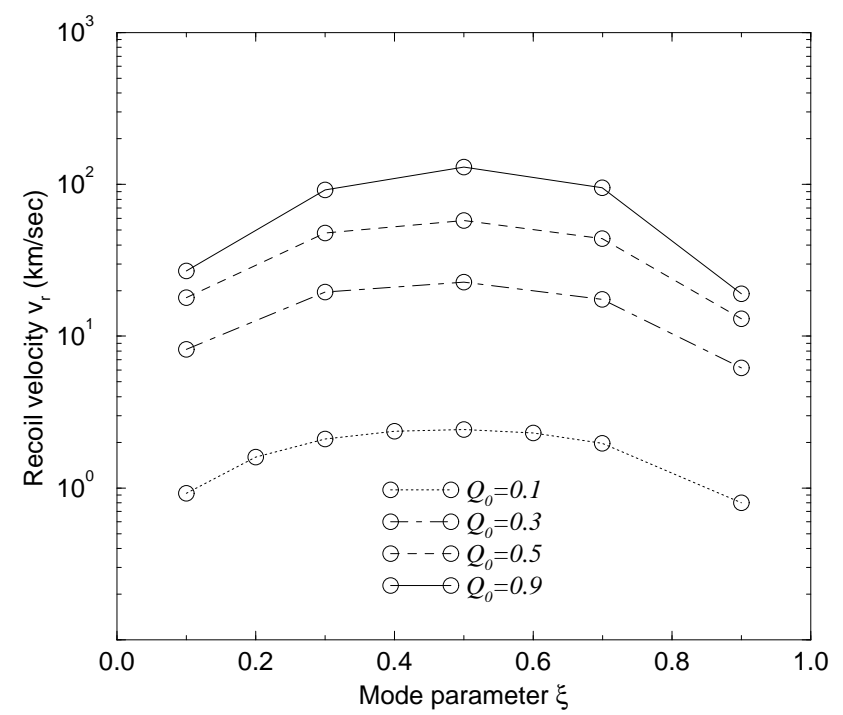

FIG. 5. Recoil velocities for even parity distortions as a function of mode parameter $\xi$ for $\eta_{0}=0$ and different wave amplitudes $Q_{0}$. The velocities are computed from the radiative momentum flux carried by gravitational waves crossing spherical shells of radius $15 M_{A D M}$ from the black hole throat.

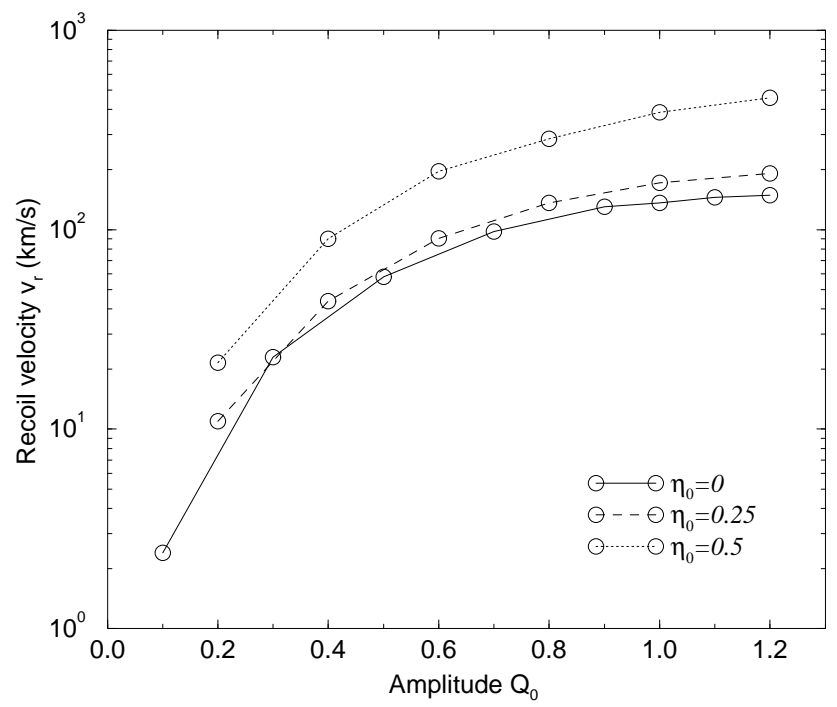

FIG. 6. Recoil velocities for even parity perturbations as a function of Brill wave amplitude $Q_{0}$ for $\xi=0.5$ and different initial wave locations $\eta_{0}$. The trend toward asymptotic flatness in the displayed curves suggests maximum recoil velocities of about 150,200 and $500 \mathrm{~km} / \mathrm{sec}$, for $\eta_{0}=0$, 0.25 and 0.5 respectively. 


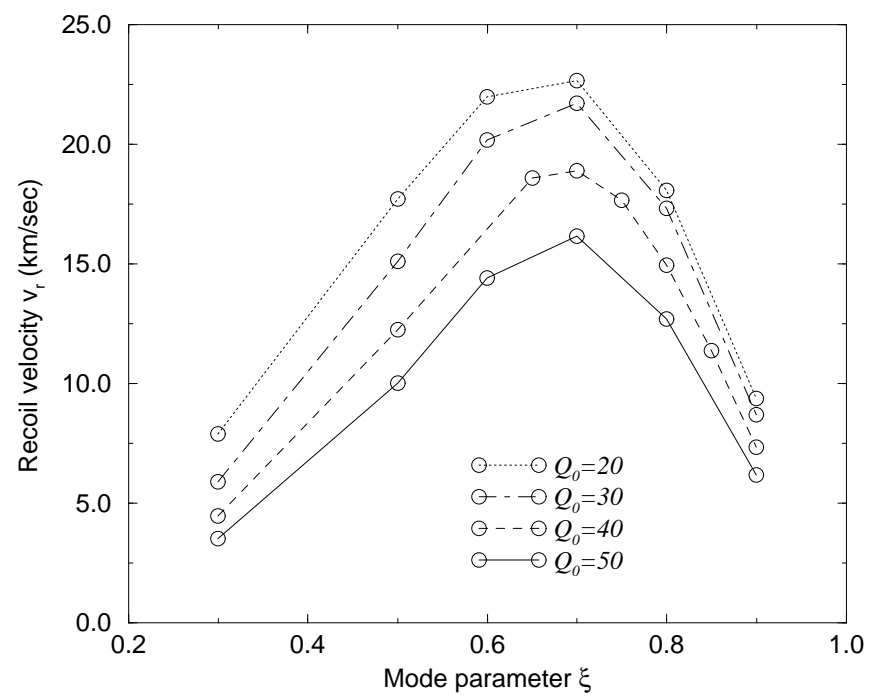

FIG. 7. Recoil velocities for odd parity distortions as a function of mode parameter $\xi$ for $\eta_{0}=0$ and different wave amplitudes $Q_{0}$.

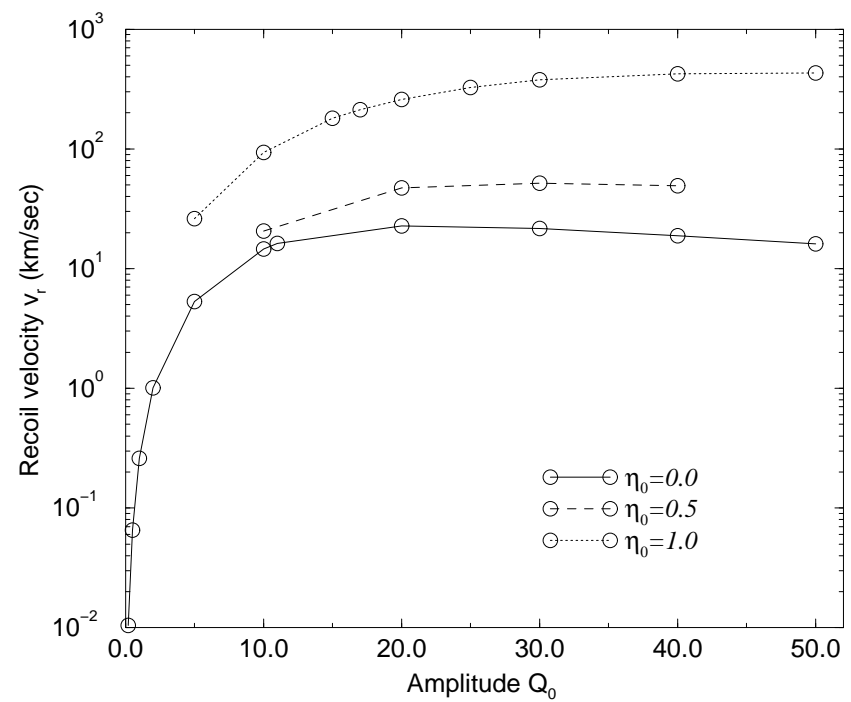

FIG. 8. Recoil velocities for odd parity perturbations as a function of Brill wave amplitude $Q_{0}$ for $\xi=0.7$ and different initial wave locations $\eta_{0}$. Maximum recoil velocities of about 23,52 and $430 \mathrm{~km} / \mathrm{sec}$ are found for $\eta_{0}=0,0.5$ and 1 respectively. 